Supplementary Data

\title{
Steric and Electrostatic Effects in DNA Synthesis by the SOS-Induced DNA Polymerases II and IV of Escherichia coli
}

\author{
Adam P. Silverman, ${ }^{1}$ Qingfei Jiang, ${ }^{2}$ Myron F. Goodman*, ${ }^{2}$ Eric T. Kool*1 \\ ${ }^{I}$ Department of Chemistry, Stanford University, Stanford, CA 94305-5080 \\ ${ }^{2}$ Department of Biological Sciences, University of Southern California, Los Angeles, CA \\ 90089-2910
}

\section{CONTENTS}

Figures S1 and S2. Representative gel data showing incorporation of unnatural dNTPs opposite an "A" template with Pol II and Pol IV.

Tables S1-S13. Representative incorporation data and Hanes-Woolf plots for Pols II and IV. 


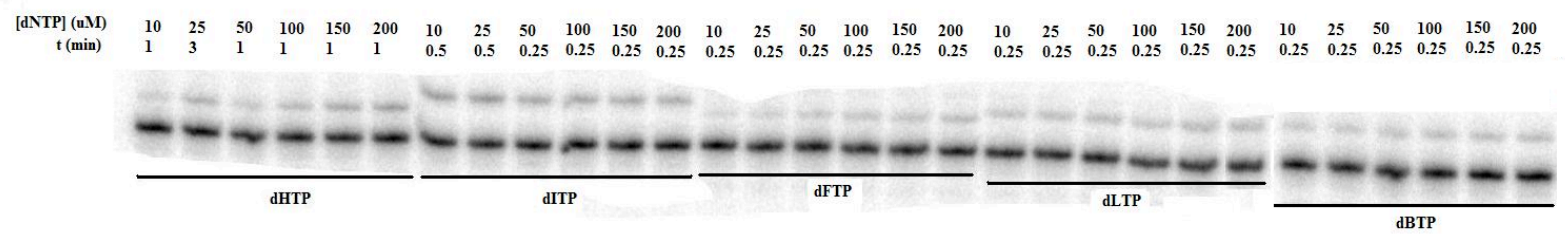

Figure S1. Gel showing incorporation of unnatural dNTPs opposite an "A" template with Pol II. Enzyme (200 nM for dHTP and dITP, $100 \mathrm{nM}$ for other samples) was incubated with the indicated concentration of dNTP, and the reaction was quenched with stop buffer after the indicated time point. 


\begin{tabular}{|c|c|c|c|c|c|}
\hline \multirow{2}{*}{$\frac{\%}{\text { incorporation }}$} & \multicolumn{3}{|c|}{ enzyme } & \multirow{2}{*}{$\frac{\text { Normalized }}{\text { Incorporation }}$} & \multirow[b]{2}{*}[\mathrm{dNTP}]{$/ \mathbf{v}$} \\
\hline & time & multiplier & [dNTP] & & \\
\hline 0.101238 & 5 & 0.5 & 10 & 0.010124 & 987.774 \\
\hline 0.182822 & 5 & 0.5 & 25 & 0.018282 & 1367.454 \\
\hline 0.111259 & 2 & 0.5 & 50 & 0.027815 & 1797.613 \\
\hline 0.184595 & 2 & 0.5 & 100 & 0.046149 & 2166.901 \\
\hline 0.252109 & 2 & 0.5 & 150 & 0.063027 & 2379.924 \\
\hline 0.278284 & 2 & 0.5 & 200 & 0.069571 & 2874.76 \\
\hline Vmax & 0.111849 & $\% / \min$ & & & \\
\hline $\mathrm{Km}$ & 126.6022 & $\mathrm{uM}$ & & & \\
\hline Efficiency & 883.4703 & Vmax/Km & & & \\
\hline
\end{tabular}

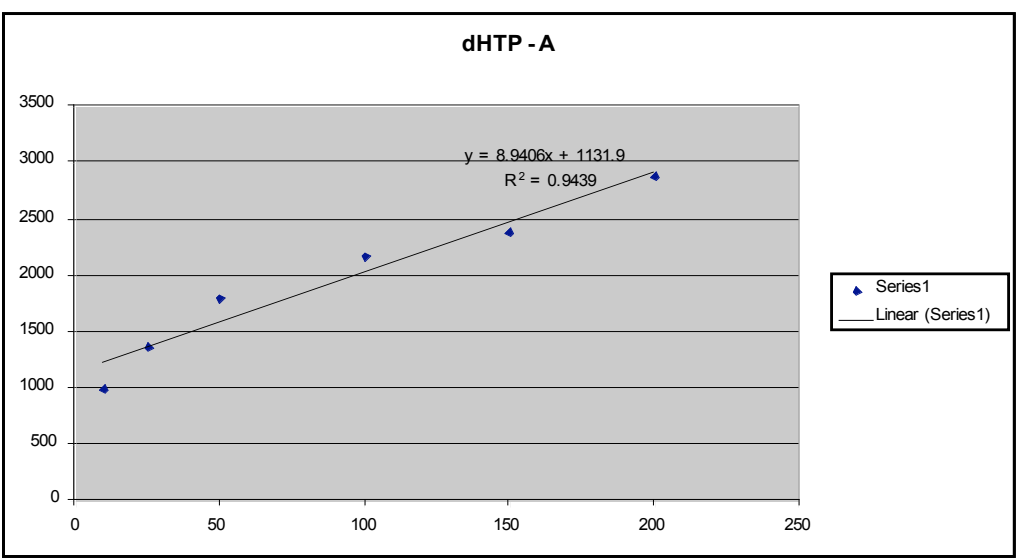

Table S1. Incorporation of dHTP opposite "A" template with Pol II. Normalized incorporation is \% incorporation times the enzyme multiplier (correction factor for different enzyme concentrations used in experiments) divided by time. The corresponding Hanes-Wolf plot from which values for Vmax and Km were derived is also shown. 


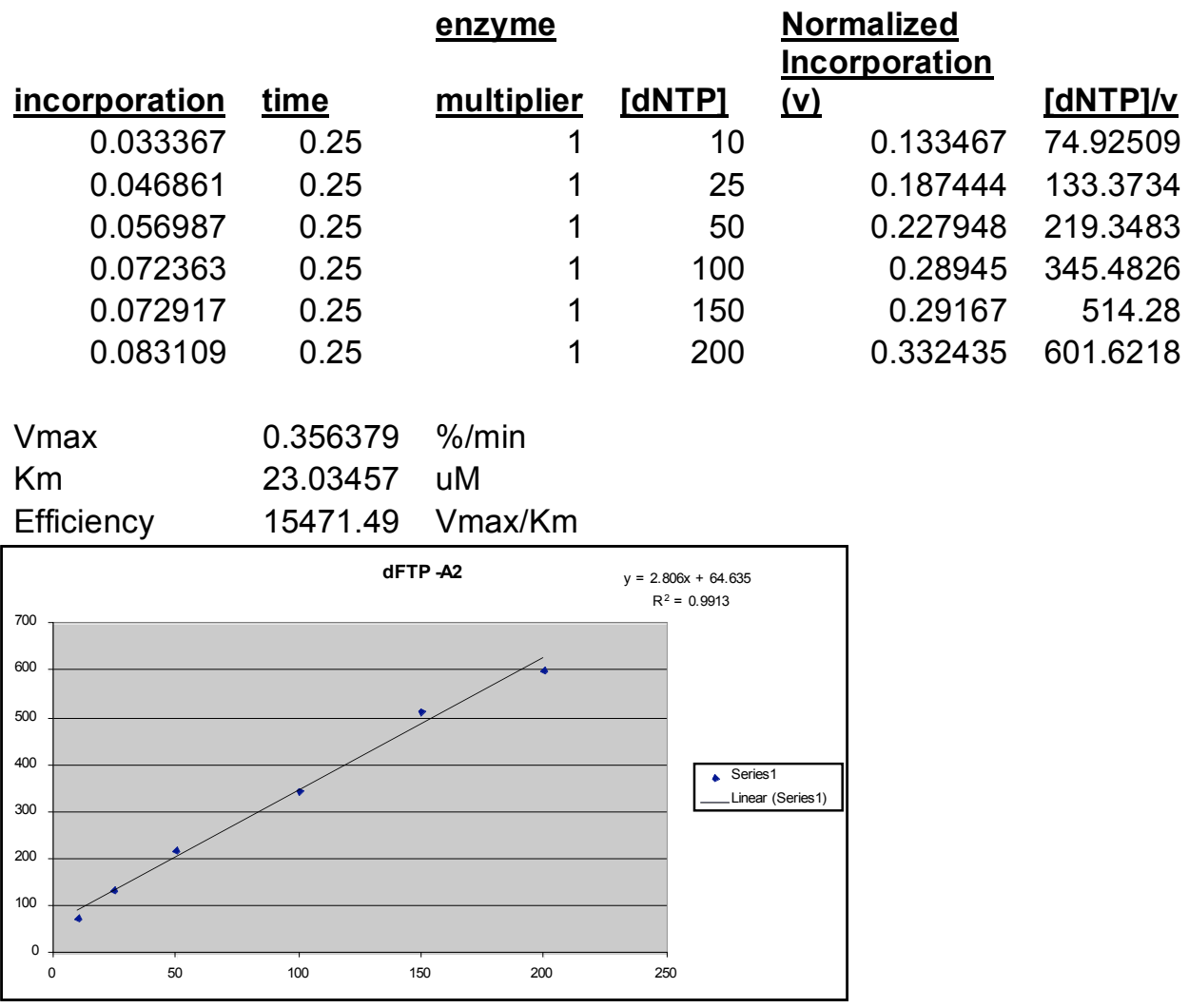

Table S2. Incorporation of dFTP opposite "A" template with Pol II. Normalized incorporation is \% incorporation times the enzyme multiplier (correction factor for different enzyme concentrations used in experiments) divided by time. The corresponding Hanes-Wolf plot from which values for Vmax and $\mathrm{Km}$ were derived is also shown. 


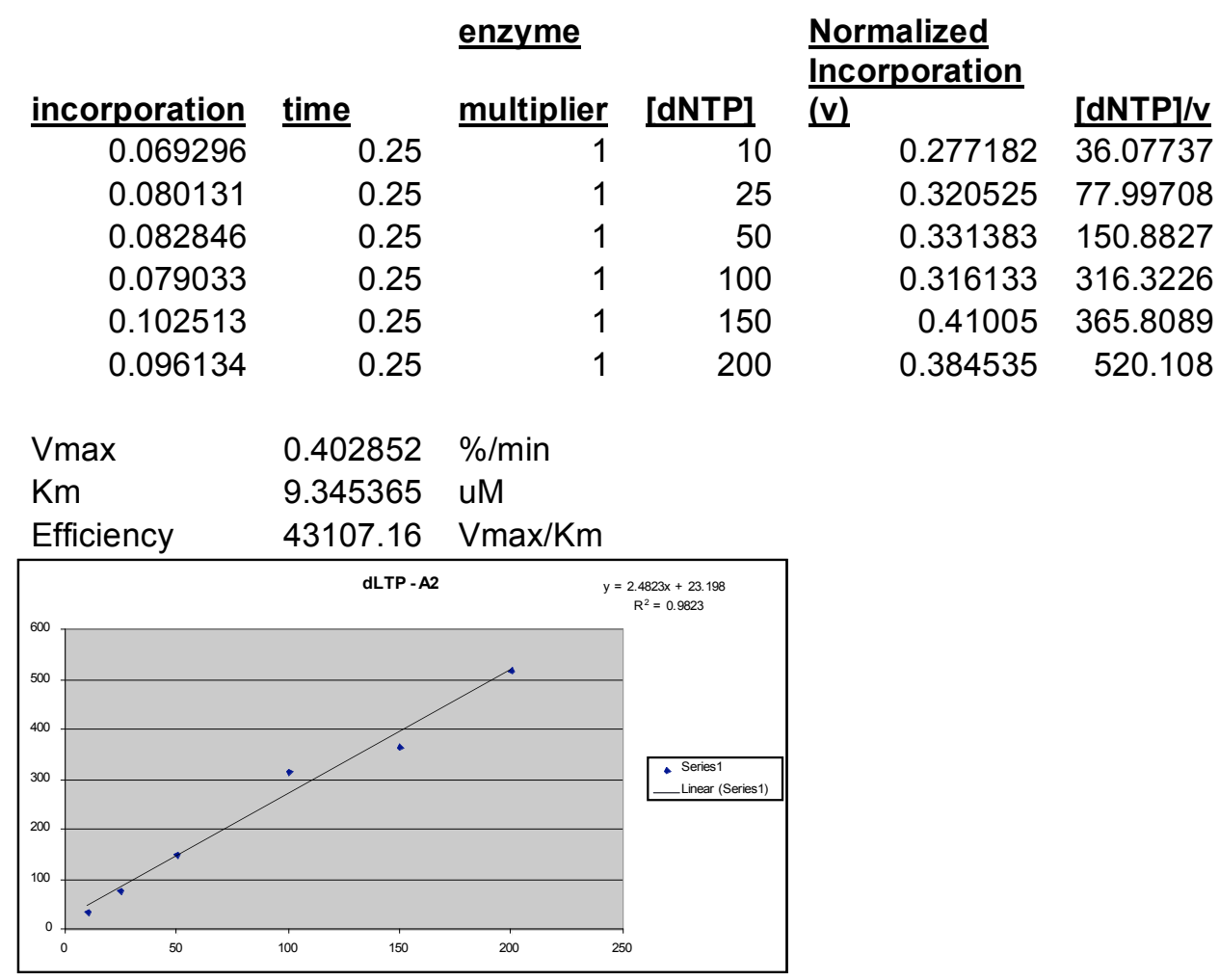

Table S3. Incorporation of dLTP opposite "A" template with Pol II. Normalized incorporation is \% incorporation times the enzyme multiplier (correction factor for different enzyme concentrations used in experiments) divided by time. The corresponding Hanes-Wolf plot from which values for Vmax and $\mathrm{Km}$ were derived is also shown. 


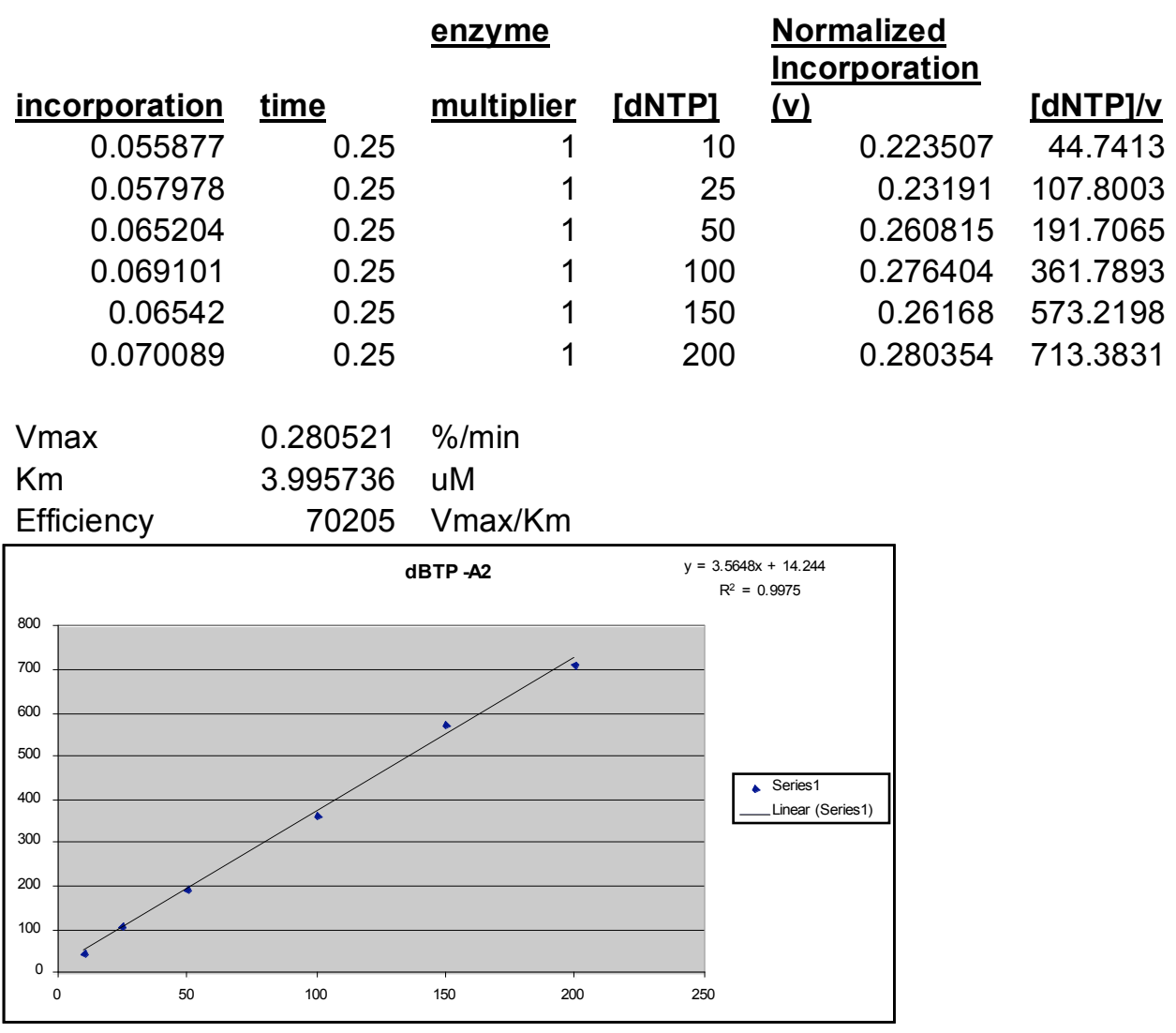

Table S4. Incorporation of dBTP opposite "A" template with Pol II. Normalized incorporation is \% incorporation times the enzyme multiplier (correction factor for different enzyme concentrations used in experiments) divided by time. The corresponding Hanes-Wolf plot from which values for Vmax and $\mathrm{Km}$ were derived is also shown. 


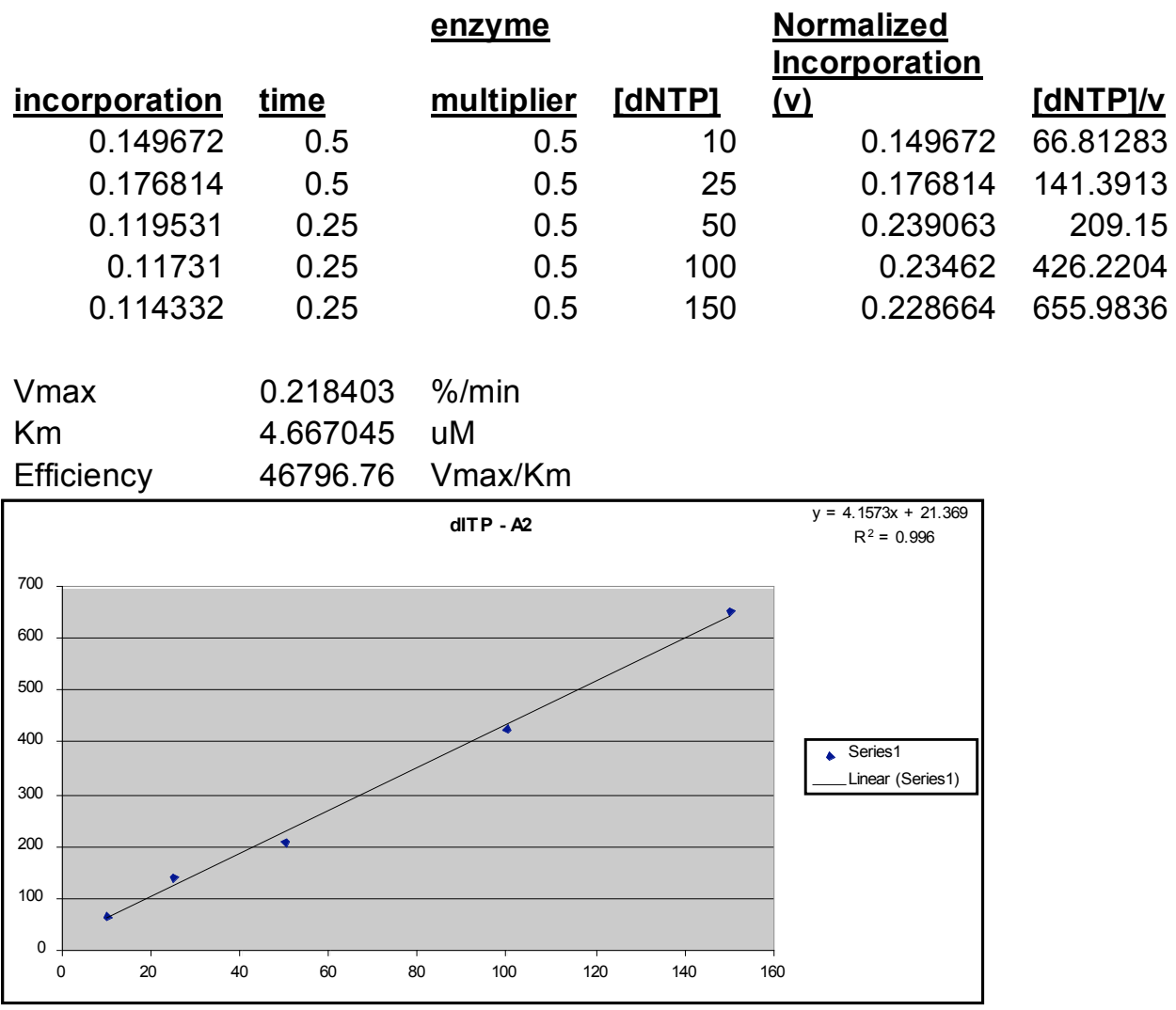

Table S5. Incorporation of dITP opposite "A" template with Pol II. Normalized incorporation is \% incorporation times the enzyme multiplier (correction factor for different enzyme concentrations used in experiments) divided by time. The corresponding Hanes-Wolf plot from which values for Vmax and $\mathrm{Km}$ were derived is also shown. 


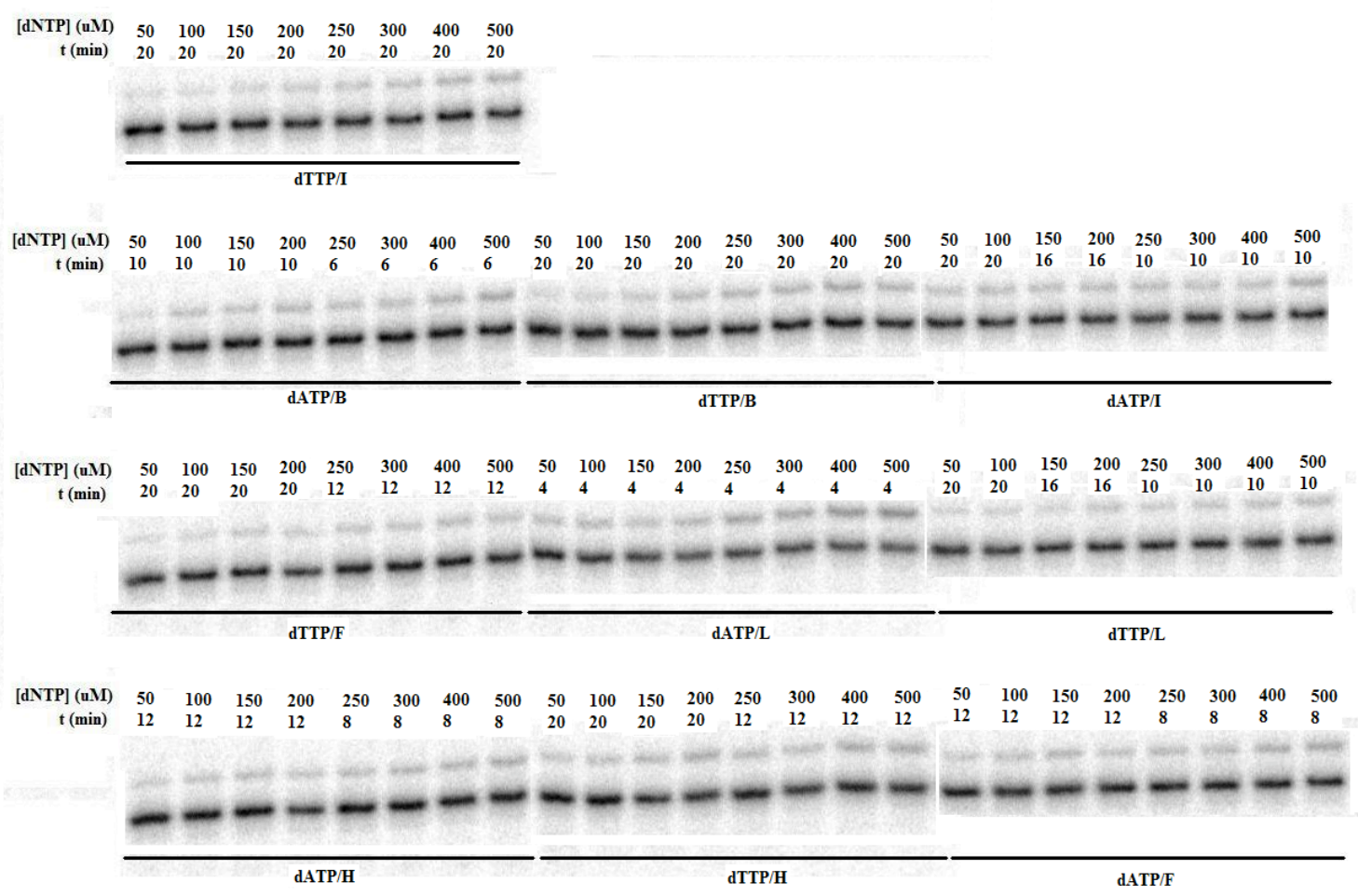

Figure S2. Gel showing incorporation of natural dATP or dTTP opposite unnatural templates, as indicated, by Pol IV. Enzyme $(1000 \mathrm{nM})$ was incubated with the indicated concentration of dNTP, and the reaction was quenched with stop buffer after the indicated time point. 


\begin{tabular}{|c|c|c|c|c|c|}
\hline & & enzyme & & $\begin{array}{l}\text { Normalized } \\
\text { Incorporation }\end{array}$ & \\
\hline incorporation & $\underline{\text { time }}$ & $\underline{\text { multiplier }}$ & [dNTP] & (v) & {$[\mathrm{dNTP}] / \mathbf{v}$} \\
\hline 0.053447 & 12 & 0.25 & 100 & 0.001113 & 89808.68 \\
\hline 0.066558 & 12 & 0.25 & 150 & 0.001387 & 108175.8 \\
\hline 0.065337 & 12 & 0.25 & 200 & 0.001361 & 146929.4 \\
\hline 0.056284 & 8 & 0.25 & 250 & 0.001759 & 142137.5 \\
\hline 0.084199 & 8 & 0.25 & 300 & 0.002631 & 114015.1 \\
\hline 0.087469 & 8 & 0.25 & 400 & 0.002733 & 146338.2 \\
\hline 0.115126 & 8 & 0.25 & 500 & 0.003598 & 138978.3 \\
\hline Vmax & 0.010137 & $\% / \min$ & & & \\
\hline $\mathrm{Km}$ & 1012.236 & $\mathrm{uM}$ & & & \\
\hline Efficiency & 10.01492 & Vmax/Km & & & \\
\hline
\end{tabular}

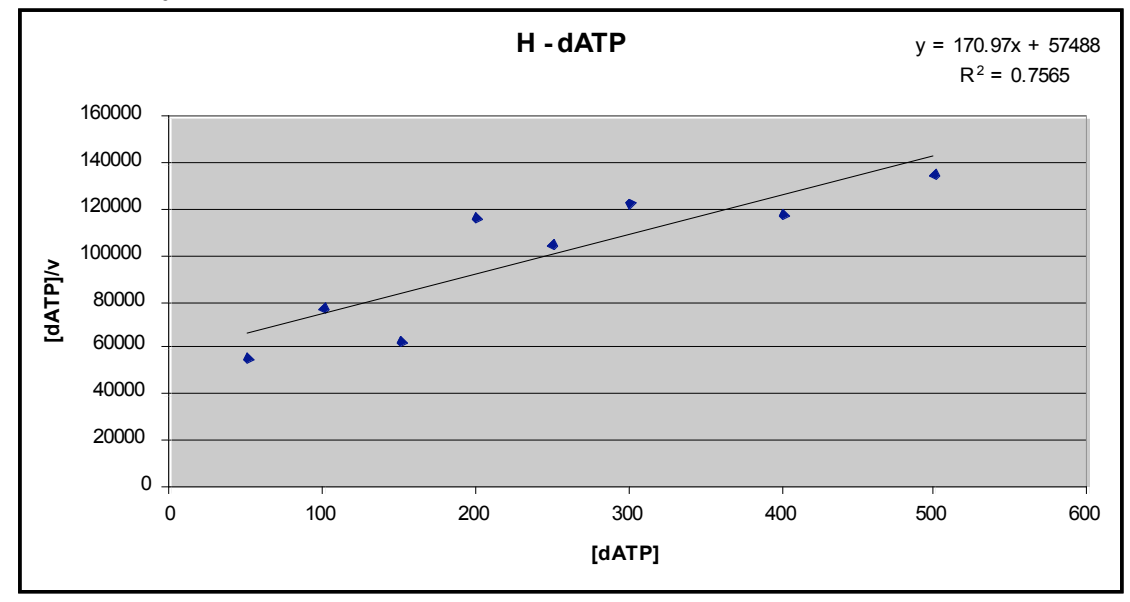

Table S6. Incorporation of dATP opposite "H" template with Pol IV. Normalized incorporation is \% incorporation times the enzyme multiplier (correction factor for different enzyme concentrations used in experiments) divided by time. The corresponding Hanes-Wolf plot from which values for Vmax and $\mathrm{Km}$ were derived is also shown. 


\begin{tabular}{|c|c|c|c|c|c|}
\hline \multirow[b]{2}{*}{ incorporation } & \multicolumn{3}{|c|}{ enzyme } & \multicolumn{2}{|l|}{$\frac{\text { Normalized }}{\text { Incorporation }}$} \\
\hline & time & multiplier & [dNTP] & & {$[\mathrm{dNTP}] / \mathrm{v}$} \\
\hline 0.052046 & 20 & 0.25 & 50 & 0.000651 & 76854.78 \\
\hline 0.097885 & 20 & 0.25 & 150 & 0.001224 & 122592.9 \\
\hline 0.140853 & 20 & 0.25 & 200 & 0.001761 & 113593.3 \\
\hline 0.083364 & 12 & 0.25 & 250 & 0.001737 & 143946.7 \\
\hline 0.085068 & 12 & 0.25 & 300 & 0.001772 & 169276.4 \\
\hline 0.117203 & 12 & 0.25 & 400 & 0.002442 & 163817.7 \\
\hline 0.139043 & 12 & 0.25 & 500 & 0.002897 & 172608.4 \\
\hline Vmax & 0.004758 & $\% / \min$ & & & \\
\hline $\mathrm{Km}$ & 390.0186 & $\mathrm{uM}$ & & & \\
\hline Efficiency & 12.19839 & Vmax/Km & & & \\
\hline
\end{tabular}

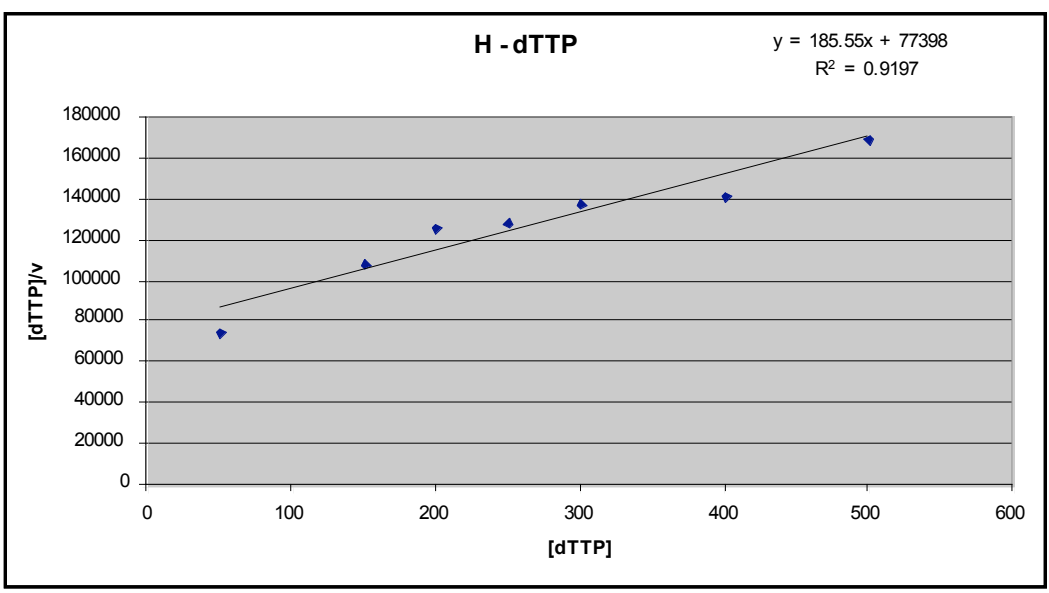

Table S7. Incorporation of dTTP opposite "H" template with Pol IV. Normalized incorporation is \% incorporation times the enzyme multiplier (correction factor for different enzyme concentrations used in experiments) divided by time. The corresponding Hanes-Wolf plot from which values for Vmax and $\mathrm{Km}$ were derived is also shown. 


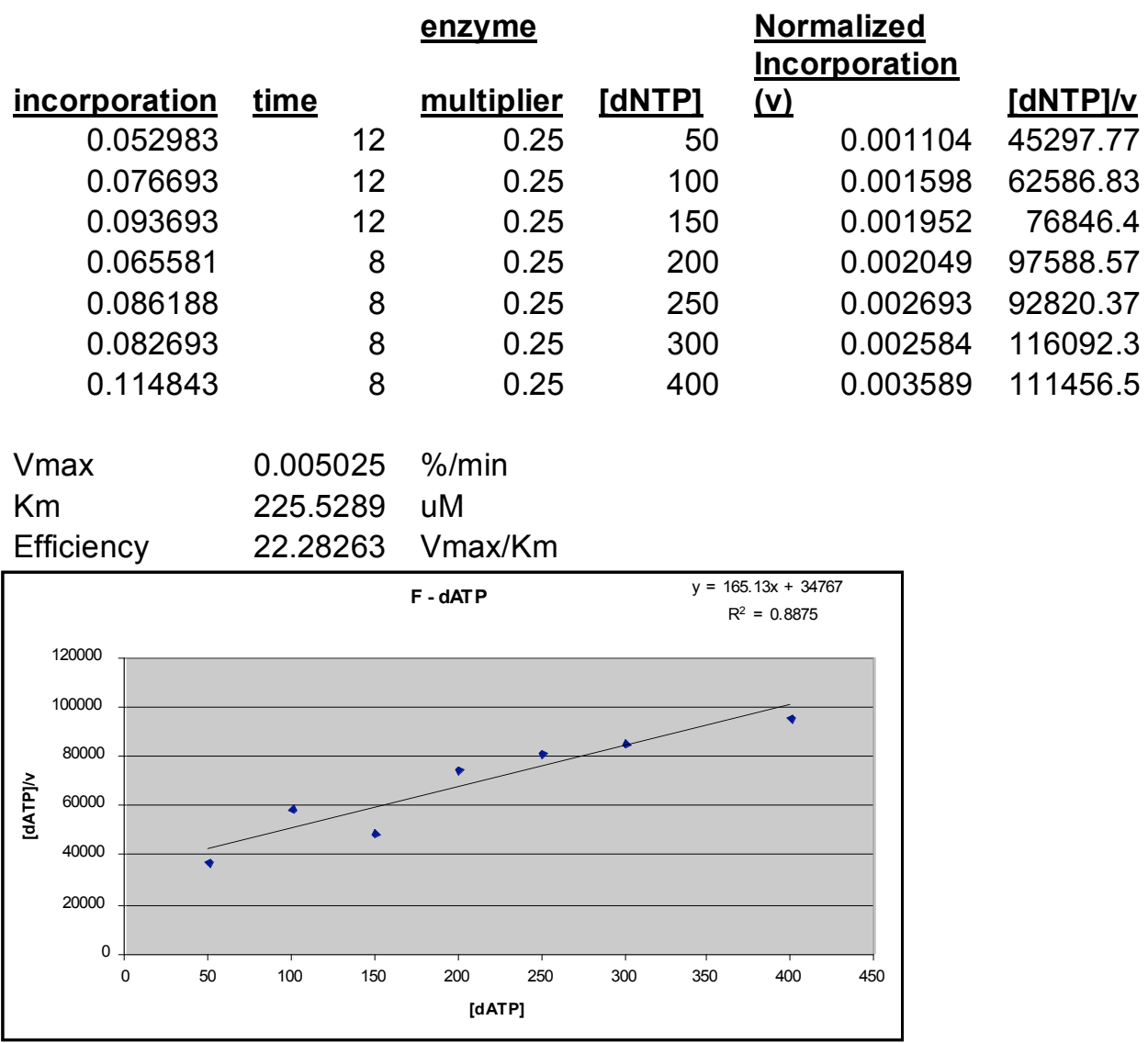

Table S8. Incorporation of dATP opposite "F" template with Pol IV. Normalized incorporation is \% incorporation times the enzyme multiplier (correction factor for different enzyme concentrations used in experiments) divided by time. The corresponding Hanes-Wolf plot from which values for Vmax and $\mathrm{Km}$ were derived is also shown. 


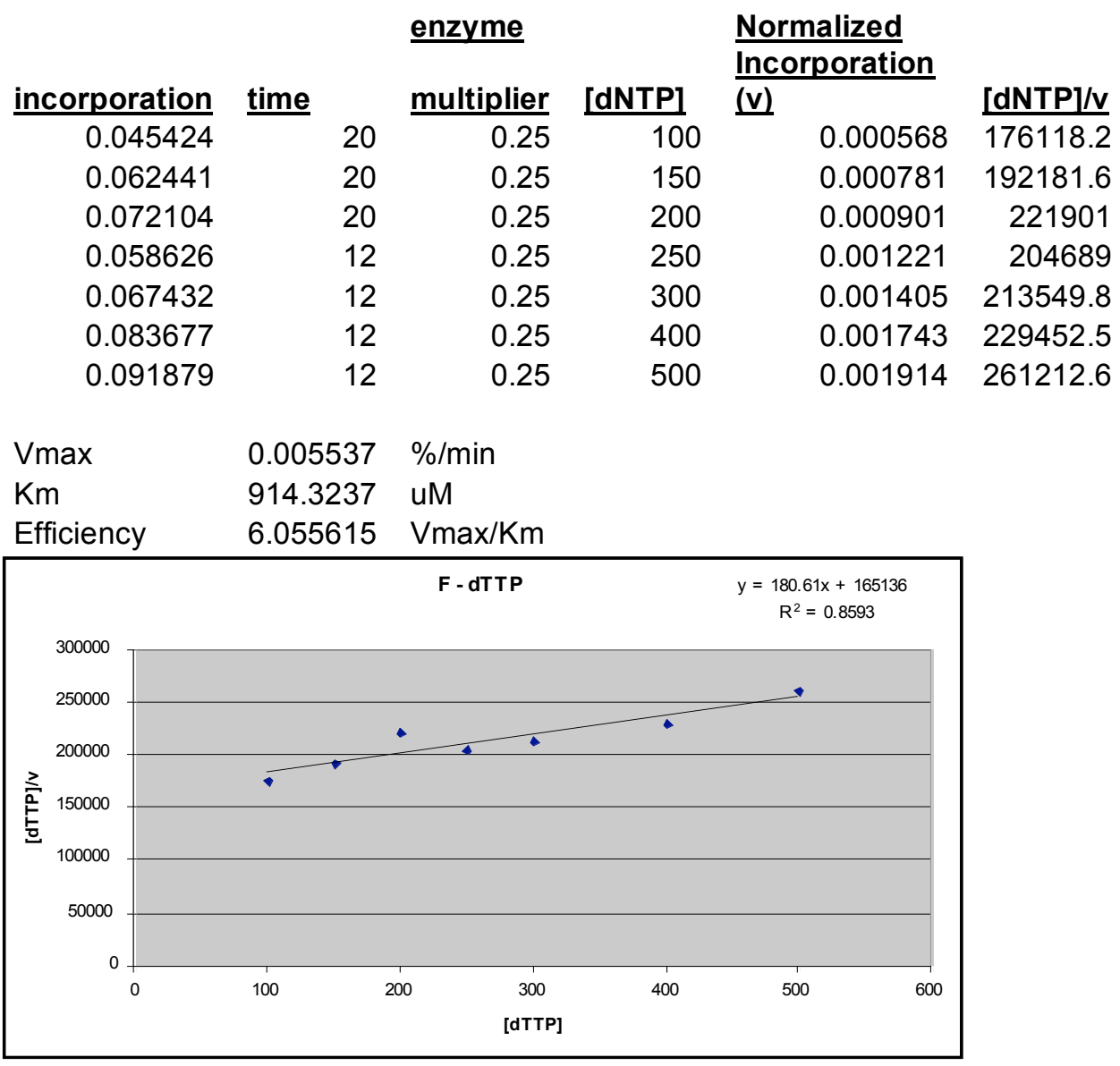

Table S9. Incorporation of dTTP opposite "F" template with Pol IV. Normalized incorporation is \% incorporation times the enzyme multiplier (correction factor for different enzyme concentrations used in experiments) divided by time. The corresponding Hanes-Wolf plot from which values for Vmax and Km were derived is also shown. 


\begin{tabular}{|c|c|c|c|c|c|}
\hline \multirow[b]{2}{*}{ incorporation } & \multicolumn{3}{|c|}{ enzyme } & \multicolumn{2}{|l|}{ Normalized } \\
\hline & time & multiplier & [dNTP] & (v) & {$[\mathrm{dNTP}] / \mathbf{v}$} \\
\hline 0.082928 & 4 & 0.25 & 50 & 0.005183 & 9646.878 \\
\hline 0.114042 & 4 & 0.25 & 100 & 0.007128 & 14029.94 \\
\hline 0.128377 & 4 & 0.25 & 150 & 0.008024 & 18694.94 \\
\hline 0.146734 & 4 & 0.25 & 200 & 0.009171 & 21808.18 \\
\hline 0.179946 & 4 & 0.25 & 250 & 0.011247 & 22228.95 \\
\hline 0.1781 & 4 & 0.25 & 300 & 0.011131 & 26951.16 \\
\hline Vmax & 0.01532 & $\% / \min$ & & & \\
\hline $\mathrm{Km}$ & 114.442 & $\mathrm{uM}$ & & & \\
\hline Efficiency & 133.8652 & Vmax/Km & & & \\
\hline
\end{tabular}

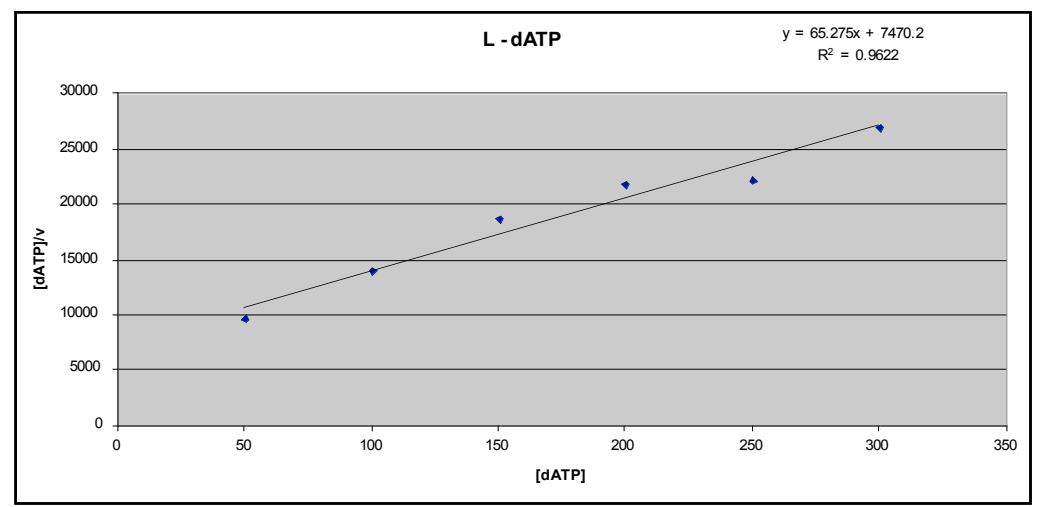

Table S10. Incorporation of dATP opposite "L" template with Pol IV. Normalized incorporation is \% incorporation times the enzyme multiplier (correction factor for different enzyme concentrations used in experiments) divided by time. The corresponding Hanes-Wolf plot from which values for Vmax and $\mathrm{Km}$ were derived is also shown. 


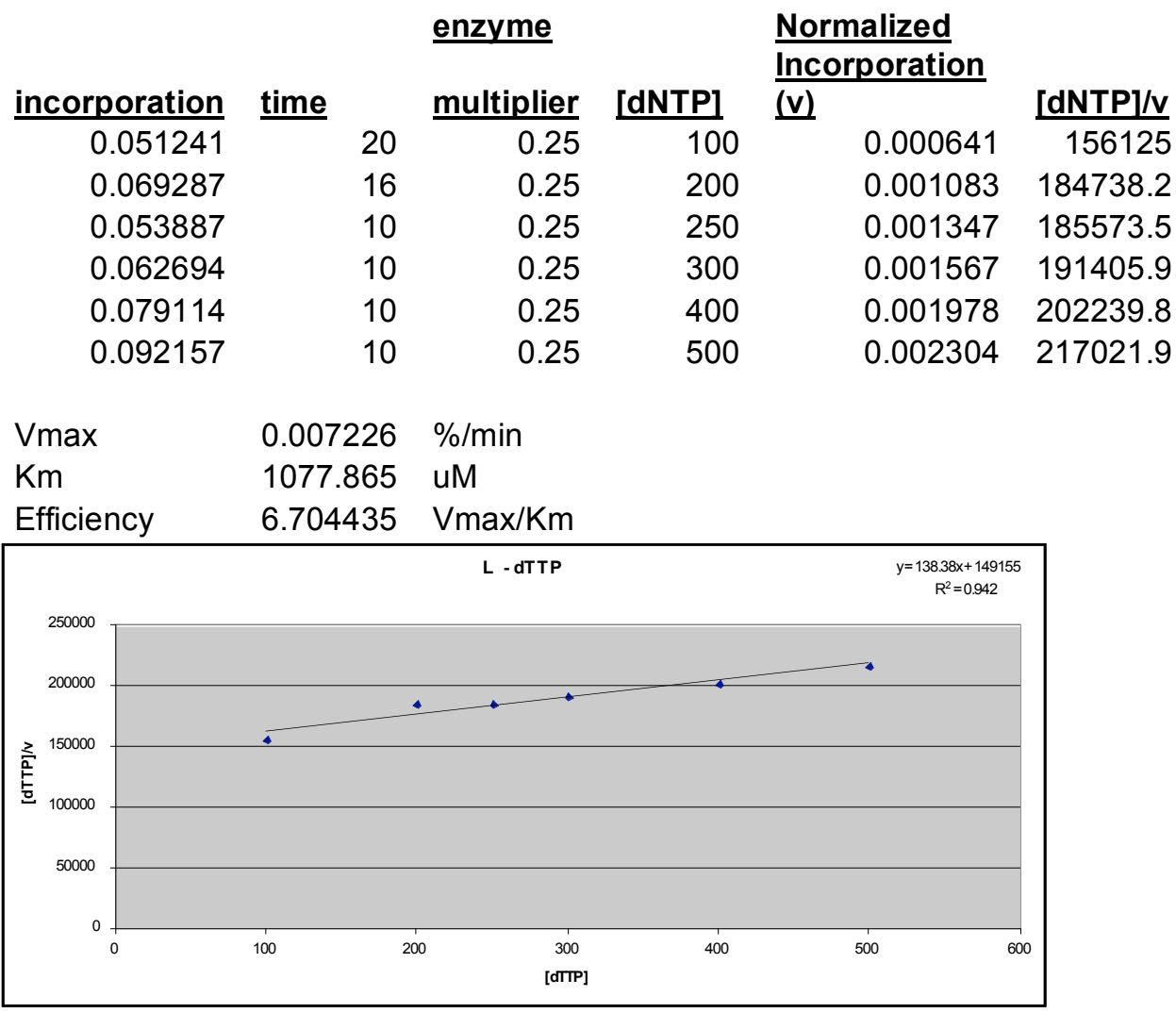

Table S11. Incorporation of dTTP opposite "L" template with Pol IV. Normalized incorporation is \% incorporation times the enzyme multiplier (correction factor for different enzyme concentrations used in experiments) divided by time. The corresponding Hanes-Wolf plot from which values for Vmax and Km were derived is also shown. 


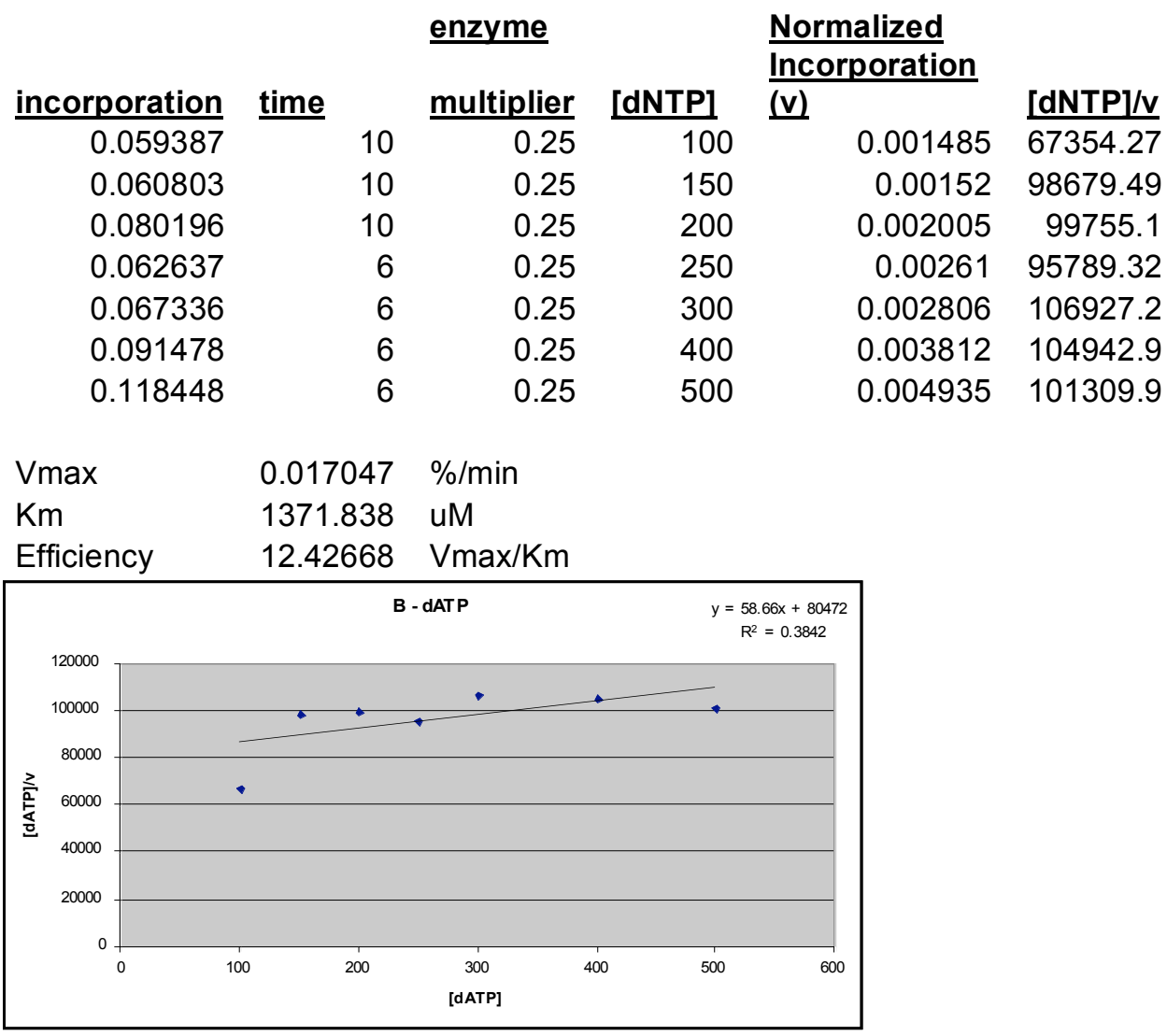

Table S12. Incorporation of dATP opposite "B" template with Pol IV. Normalized incorporation is \% incorporation times the enzyme multiplier (correction factor for different enzyme concentrations used in experiments) divided by time. The corresponding Hanes-Wolf plot from which values for Vmax and $\mathrm{Km}$ were derived is also shown. 


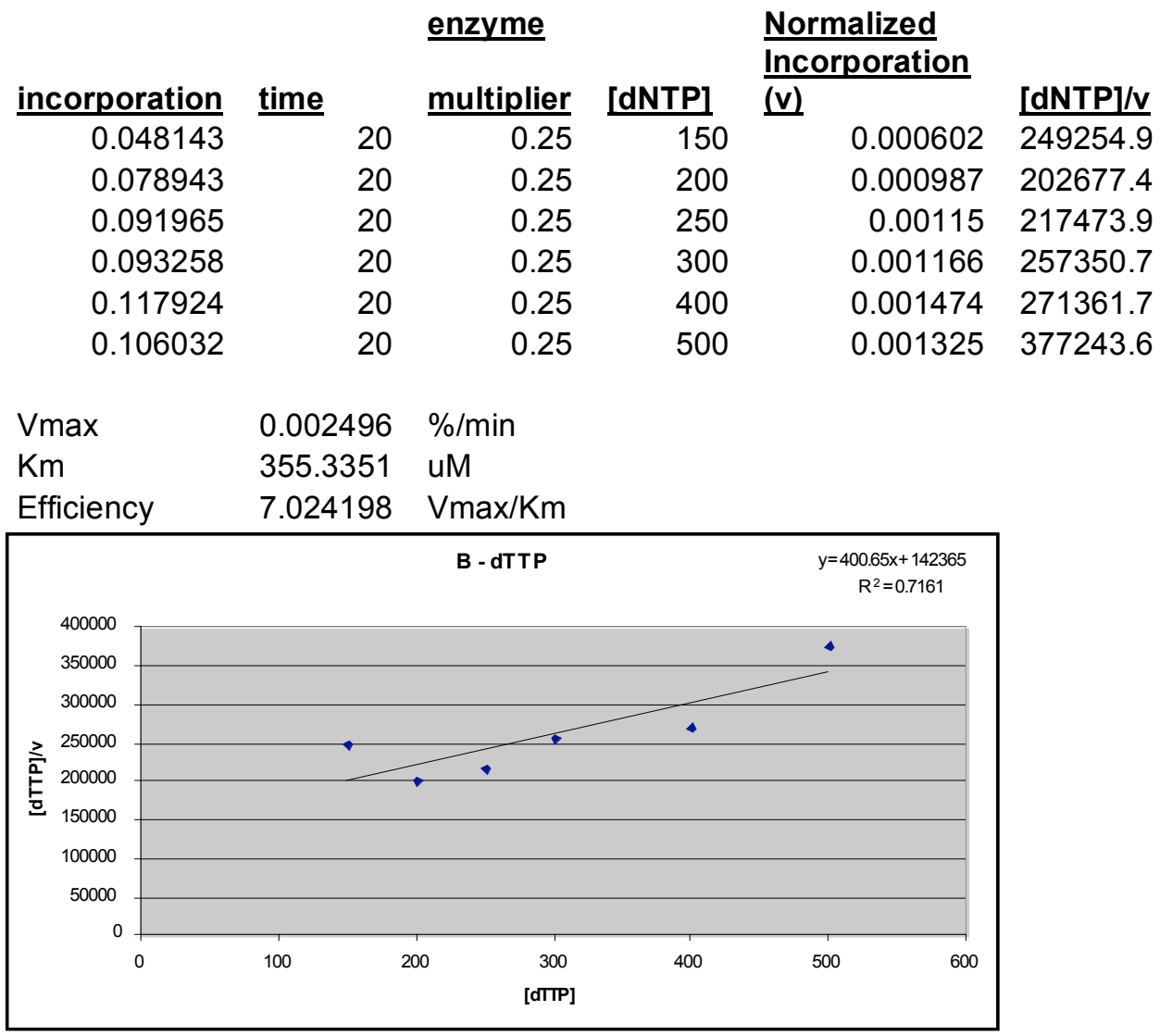

Table S13. Incorporation of dTTP opposite "B" template with Pol IV. Normalized incorporation is \% incorporation times the enzyme multiplier (correction factor for different enzyme concentrations used in experiments) divided by time. The corresponding Hanes-Wolf plot from which values for Vmax and $\mathrm{Km}$ were derived is also shown. 


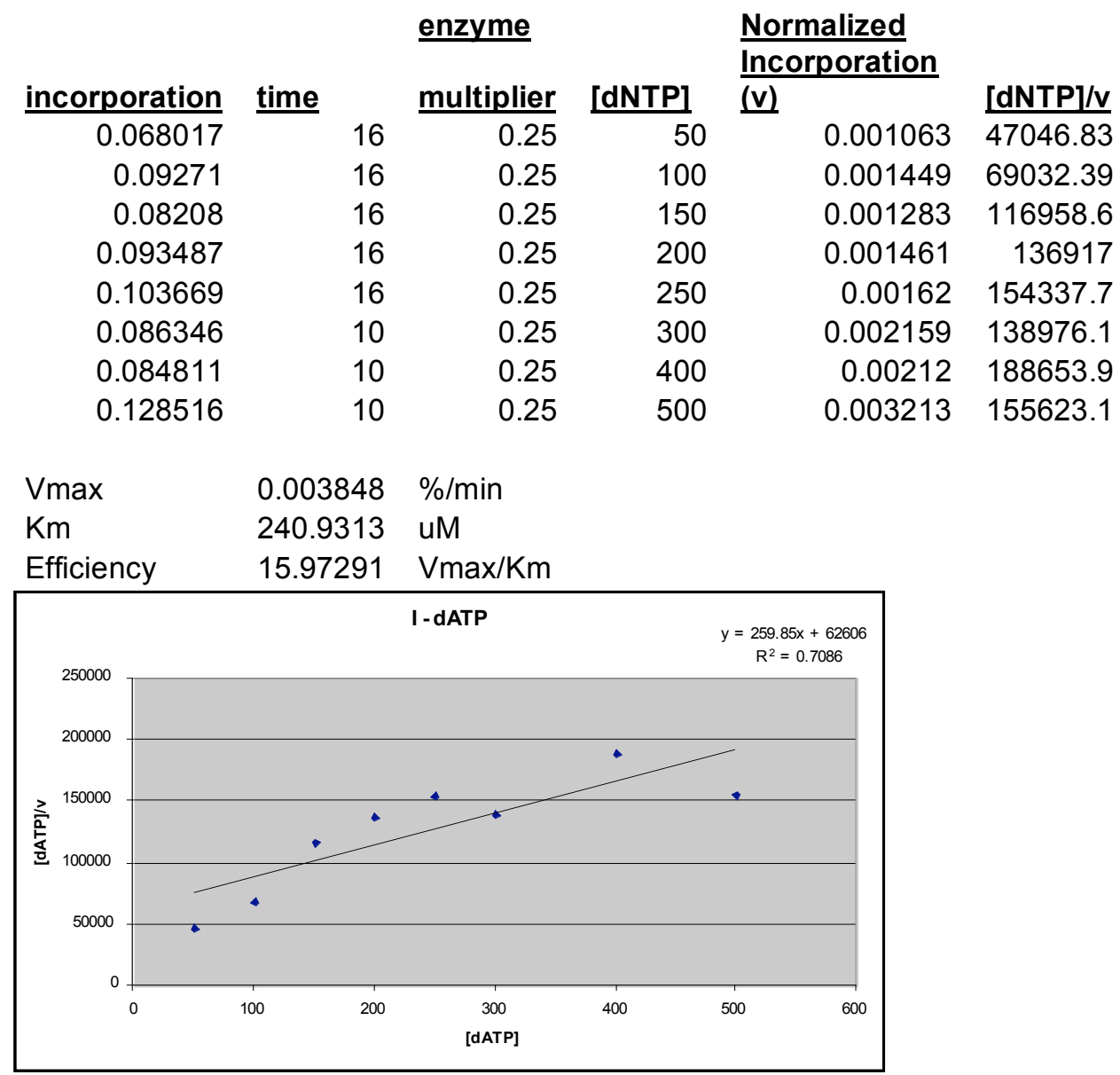

Table S13. Incorporation of dATP opposite "I" template with Pol IV. Normalized incorporation is \% incorporation times the enzyme multiplier (correction factor for different enzyme concentrations used in experiments) divided by time. The corresponding Hanes-Wolf plot from which values for Vmax and $\mathrm{Km}$ were derived is also shown. 


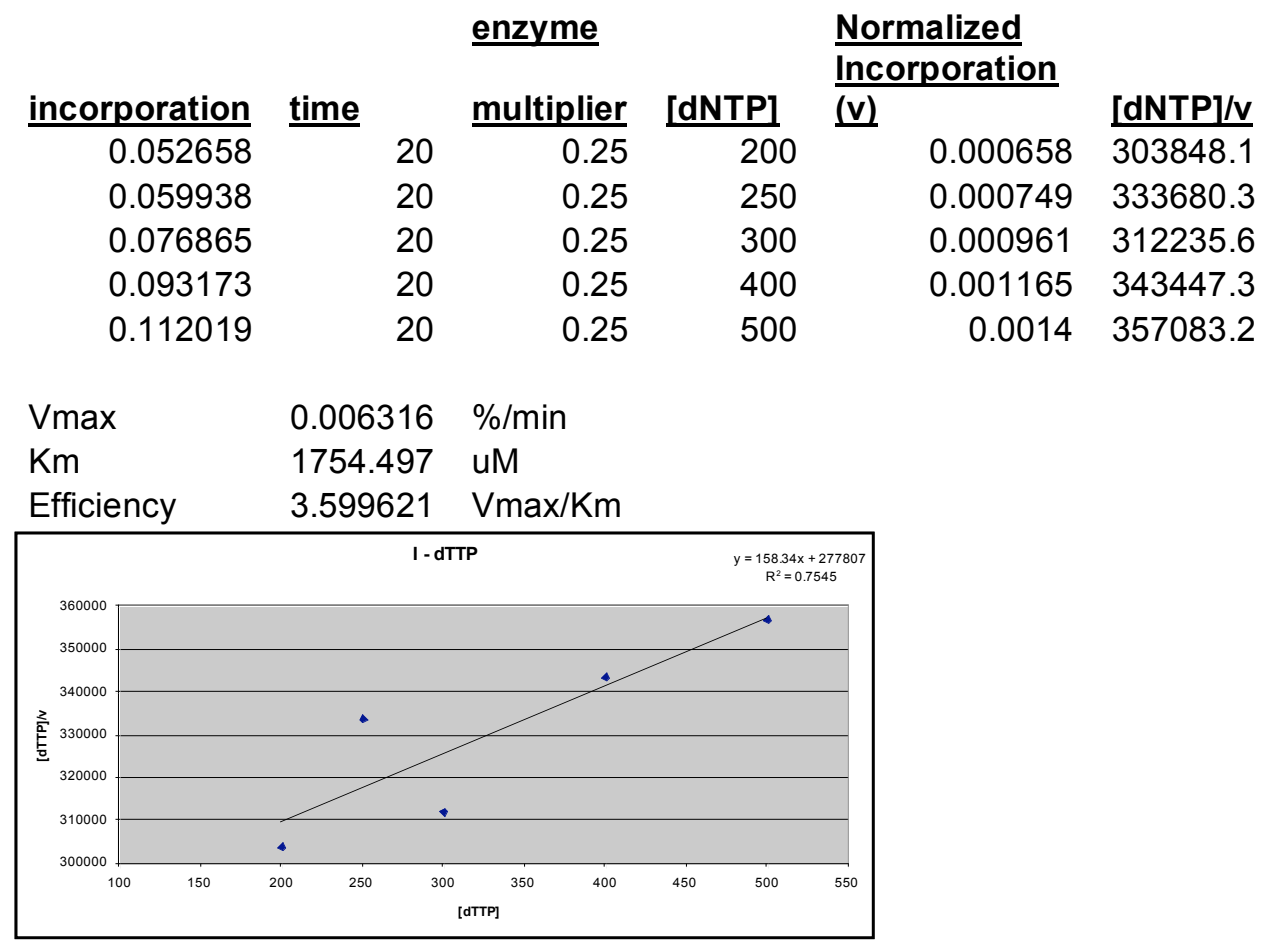

Table S13. Incorporation of dTTP opposite "I" template with Pol IV. Normalized incorporation is \% incorporation times the enzyme multiplier (correction factor for different enzyme concentrations used in experiments) divided by time. The corresponding Hanes-Wolf plot from which values for Vmax and Km were derived is also shown. 